\title{
The Herbrand Functional Interpretation of the Double Negation Shift
}

\author{
Martín Escardó Paulo Oliva \\ Preprint, SePtember 12, 2018
}

\begin{abstract}
This paper considers a generalisation of selection functions over an arbitrary strong monad $T$, as functionals of type $J_{R}^{T} X=(X \rightarrow R) \rightarrow T X$. It is assumed throughout that $R$ is a $T$-algebra. We show that $J_{R}^{T}$ is also a strong monad, and that it embeds into the continuation monad $K_{R} X=(X \rightarrow R) \rightarrow$ $R$. We use this to derive that the explicitly controlled product of $T$-selection functions is definable from the explicitly controlled product of quantifiers, and hence from Spector's bar recursion. We then prove several properties of this product in the special case when $T$ is the finite power set monad $\mathcal{P}_{\mathrm{f}}(\cdot)$. These are used to show that when $T X=\mathcal{P}_{\mathrm{f}}(X)$ the explicitly controlled product of $T$-selection functions calculates a witness to the Herbrand functional interpretation of the double negation shift.
\end{abstract}

\section{Introduction}

Gödel's functional or Dialectica interpretation was introduced in 10] as a reduction of first order arithmetic to the "finitistic" quantifier-free calculus of primitive recursive functionals (system T). Soon after Gödel's paper appeared in print, Spector [16] showed how Gödel's interpretation of arithmetic could be extended to analysis by extending system $\mathrm{T}$ with what he called bar recursion. By analysis we mean classical arithmetic in all finite types extended with countable choice and dependent choice - and hence comprehension.

Spector's original work has given rise to several other bar recursive interpretations of analysis, whereby different proof interpretations other than the Dialetica interpretation have been used. In such cases one was either able to continue using Spector's original form of bar recursion (e.g. [8, 12]) or some variant of bar recursion was proposed (e.g. [1, 2]).

As we have shown in [5, 6], there are close connections between the different forms of bar recursion and the calculation of optimal strategies in a general class of sequential games. This was achieved by showing that bar recursion turns out to correspond to the iterated product of quantifiers and selection functions. Spector's original bar recursion can be shown to be equivalent to the iterated product of quantifiers, whereas the restricted form needed to witness the Dialectica interpretation of DNS is equivalent to the iterated product of selection functions 7 . 
This analogy between computability and games is based on the modelling of players via quantifiers $K_{R} X=(X \rightarrow R) \rightarrow R$. If $X$ is the set of moves available to a player, and $R$ is the set of possible outcomes, then mappings of type $X \rightarrow R$ can be seen as describing the context a player lives in. Such contexts (a form of continuation) describe the final outcome for each of the possible choices of the player. Hence, to specify a player is to describe her preferred outcomes for each given game context. Similarly, a selection function $J_{R} X=(X \rightarrow R) \rightarrow X$ also takes a game context as input, but determines the optimal move for any given game context.

In this paper we consider the iterated product of selection functions parametrised by an arbitrary strong monad $T X$, i.e. $J_{R}^{T} X=(X \rightarrow R) \rightarrow T X$. Using the intuition that an element of a monad $T X$ provides "information" about concrete elements of $X$, and the correspondence with games, we can view such selection functions $J_{R}^{T} X$ as specifying some information about the optimal move for any given game context.

We study the bar recursion that arises from the iterated product of such $T$ selection functions. Our first step is to show that $J_{R}^{T} X$ is also a strong monad. Since any strong monad embeds into the continuation monad, it follows that we have an embedding of $J_{R}^{T} X$ into $K X$. We make use of this embedding to show that the iterated product of $T$-selection functions is in fact primitive recursively definable from the iterated product of quantifiers, and hence from Spector's original bar recursion.

Finally, we consider the particular case when $T X$ is the finite power set monad $\mathcal{P}_{\mathrm{f}}(X)$. We prove several properties of the iterated product of selection functions $(X \rightarrow R) \rightarrow \mathcal{P}_{\mathrm{f}}(X)$, and show how it provides a witness for the Herbrand functional interpretation [18] of double-negation shift DNS

$$
\forall n^{\mathbb{N}} \neg \neg A(n) \rightarrow \neg \neg \forall n^{\mathbb{N}} A(n) .
$$

\subsection{Heyting arithmetic in all finite types, and bar induction}

We work in the setting of Heyting arithmetic in all finite types, with full extensionality. This corresponds to the system E-HA ${ }^{\omega}$ of 17 . When carrying out the verification of the Herbrand functional interpretation of DNS we will make free use of classical logic, in order to simplify the verification of the bar-recursive construction, hence will be working on E-PA ${ }^{\omega}$. Although it is well-known that full extensionality is not normally interpreted by the functional interpretations, we are simply assuming full extensionality in the verification of our interpretation of DNS, which is obviously harmless.

The quantifier-free part of the theories E-HA ${ }^{\omega}$ and E-PA ${ }^{\omega}$ is normally referred to as Gödel's system T. Although in T one normally only assumes the natural numbers $\mathbb{N}$ as basic types, and function space constructions $X \rightarrow Y$ as the only type constructor, we will follow here the same formulation of $T$ as in [18] where one also assumes products $X \times Y$, finite sequences $X^{*}$, and even finite power sets $\mathcal{P}_{\mathrm{f}}(X)$. We write $r \preceq s$ to say that the finite sequence $r$ is a prefix of the finite sequence $s$. We assume that each type $X$ contains a 'default' value $\mathbf{0}: X$, so that we 
can define an canonical extension operation $(\cdot)^{+}: X^{*} \rightarrow X^{\mathbb{N}}$ from finite to infinite sequences, by appending an infinite sequence of default values. For instance, for the natural numbers $\mathbf{0}^{\mathbb{N}}$ could be the number zero, whereas for $\mathcal{P}_{\mathrm{f}}(X)$ we can take $\mathbf{0}^{\mathcal{P}_{\mathrm{f}}(X)}=\emptyset$.

On top of E-HA ${ }^{\omega}$, in the proofs of Lemmas 3.2 and 3.3 will make use of the following form of bar induction:

Definition 1.1 (Bar induction) Let $P(s)$ be a universal formula, and $s: X^{*}$. We say that bar induction holds for $P(s)$ if whenever

- $\omega\left(s^{+}\right)<|s|$ implies $P(s)$, and

- $\omega\left(s^{+}\right) \geq|s|$ and $\forall x P(s * x)$ implies $P(s)$

then $P(\langle\rangle)$.

This form of bar induction implicitly assumes that the bar condition $\omega\left(s^{+}\right)<|s|$ eventually holds. This is indeed the case in all models of Spector's bar recursion [3, 15].

Notation. In the paper we will use sub-scripts in four different ways, and hope their respective meanings will be clear from context:

- In the following section we use sub-scripts to denote the type of a functional. For instance, the identity function of type $X$ will be written as $\operatorname{id}_{X}$.

- If $\alpha: X \rightarrow Y$ we can view $\alpha$ as a family of elements of $Y$ indexed by $X$, i.e. $\left\{\alpha_{x}\right\}_{x: X}$. When taking this view we might write $\alpha_{x}$ instead of $\alpha(x)$.

- Bar recursive functionals have several parameters, normally $\operatorname{BR}(\omega)(s)(\varepsilon)(q)$. In order to focus on the selection functions $\varepsilon$ and the outcome function $q$ we shall rewrite this as $\mathrm{BR}_{s}^{\omega}(\varepsilon)(q)$. This makes sense since $s$ is the 'index' of the bar recursion whereas $\omega$ is the stopping condition.

- Finally, for $q: X^{*} \rightarrow R$ we write $q_{s}(t)$ for $q(s * t)$ when we wish to 'partially evaluate' $q$ on $s$ to produce another function $q_{s}: X^{*} \rightarrow R$.

\subsection{Strong monads}

In this section we recall the basic notions about strong monads needed in this paper. Throughout the paper we work in Gödel's system T. Hence, $X, Y$ and $R$ should be viewed as finite types 1 .

Definition 1.2 (Strong monad) Let $T$ be a meta-level unary operation on simple types, that we will call a type operator. A type operator $T$ is called a strong monad if we have a family of closed terms

\footnotetext{
${ }^{1}$ It will be clear, however, that what we describe would work more generally in any of the well-known models of higher-order computability.
} 


$$
\begin{aligned}
\eta_{X} & : X \rightarrow T X \\
(\cdot)^{\dagger} & :(X \rightarrow T Y) \rightarrow(T X \rightarrow T Y)
\end{aligned}
$$

satisfying (provably in $\mathrm{T}$ ) the laws

(i) $\left(\eta_{X}\right)^{\dagger}=\operatorname{id}_{T X}$

(ii) $g^{\dagger} \circ \eta_{Y}=g$

(iii) $\left(g^{\dagger} \circ f\right)^{\dagger}=g^{\dagger} \circ f^{\dagger}$

where $g: Y \rightarrow T R$ and $f: X \rightarrow T Y$. When several strong monads are involved we shall use the super-script in $\eta^{T}$ so as to be clear which $\eta$ is being used.

Given $f: X \rightarrow Y$ we define $T f: T X \rightarrow T Y$ by $T f=\left(\eta_{Y} \circ f\right)^{\dagger}$. The laws for the monad show that this construction makes $T$ into a functor, that is, $T \mathrm{id}_{X}=\mathrm{id}_{T X}$, and for $g: Y \rightarrow Z$ we have $T(g \circ f)=T g \circ T f$.

Monads have been extensively studied in category theory [11, programming language semantics [13, and in the functional programming community [19]. In a monad one would normally have a non-uniform mapping from $f: X \rightarrow T Y$ to $f^{\dagger}: T X \rightarrow T Y$. The term strong here refers to the assumption that we have a uniform map $(\cdot)^{\dagger}:(X \rightarrow T Y) \rightarrow(T X \rightarrow T Y)$.

Definition 1.3 (T-algebra) Given a strong monad $T$, a type $R$ is called a $T$ algebra if we have a family of maps $(\cdot)^{*}:(X \rightarrow R) \rightarrow(T X \rightarrow R)$ satisfying

(i) $g^{*} \circ \eta_{Y}=g$

(ii) $\left(g^{*} \circ f\right)^{*}=g^{*} \circ f^{\dagger}$

where $g: Y \rightarrow R$ and $f: X \rightarrow T Y$.

The reason we focus here on strong monads is that on such monads we can define a binary product operation as follows:

Lemma 1.4 For any strong monad $T$ we can define a product operation

$$
\otimes: T X \times(X \rightarrow T Y) \rightarrow T(X \times Y)
$$

as

$$
a \otimes f=\left(\lambda x \cdot\left(\lambda y \cdot \eta_{X \times Y}(x, y)\right)^{\dagger}(f x)\right)^{\dagger}(a)
$$

satisfying, for $q: X \times Y \rightarrow T R$,

$$
q^{\dagger}(a \otimes f)=\left(\lambda x \cdot\left(q_{x}\right)^{\dagger}(f x)\right)^{\dagger}(a)
$$

where $q_{x}=\lambda y \cdot q(x, y)$. When $q: X \times Y \rightarrow R$ and $R$ is a T-algebra it satisfies

$$
q^{*}(a \otimes f)=\left(\lambda x \cdot\left(q_{x}\right)^{*}(f x)\right)^{*}(a) .
$$


Proof. We calculate as follows:

$$
\begin{aligned}
& q^{\dagger}(a \otimes f) \quad \stackrel{1}{=} \quad q^{\dagger}\left(\left(\lambda x \cdot\left(\lambda y \cdot \eta_{X \times Y}(x, y)\right)^{\dagger}(f x)\right)^{\dagger}(a)\right) \\
& \stackrel{(\circ)}{=} \quad\left(q^{\dagger} \circ\left(\lambda x \cdot\left(\lambda y \cdot \eta_{X \times Y}(x, y)\right)^{\dagger}(f x)\right)^{\dagger}\right)(a) \\
& \mathrm{D} \underline{1.2}_{=}^{i i i)} \quad\left(q^{\dagger} \circ\left(\lambda x \cdot\left(\lambda y \cdot \eta_{X \times Y}(x, y)\right)^{\dagger}(f x)\right)\right)^{\dagger}(a) \\
& \stackrel{(\circ)}{=} \quad\left(\lambda x \cdot q^{\dagger}\left(\left(\lambda y \cdot \eta_{X \times Y}(x, y)\right)^{\dagger}(f x)\right)\right)^{\dagger}(a) \\
& \stackrel{(\circ)}{=} \quad\left(\lambda x \cdot\left(\left(q^{\dagger} \circ\left(\lambda y \cdot \eta_{X \times Y}(x, y)\right)^{\dagger}\right)(f x)\right)\right)^{\dagger}(a) \\
& \mathrm{D}{ }^{1.2}{ }^{i i i)} \quad\left(\lambda x .\left(\left(q^{\dagger} \circ\left(\lambda y \cdot \eta_{X \times Y}(x, y)\right)\right)^{\dagger}(f x)\right)\right)^{\dagger}(a) \\
& \stackrel{(\circ)}{=} \quad\left(\lambda x \cdot\left(\left(\lambda y \cdot q^{\dagger}\left(\eta_{X \times Y}(x, y)\right)\right)^{\dagger}(f x)\right)\right)^{\dagger}(a) \\
& \mathrm{D}^{\underline{1.2}} \text { ii } \quad\left(\lambda x \cdot\left(q_{x}\right)^{\dagger}(f x)\right)^{\dagger}(a) \text {. }
\end{aligned}
$$

In the case $q: X \times Y \rightarrow R$ and $R$ is a $T$-algebra we use properties $(i)$ and $(i i)$ of Definition 1.3 instead.

\section{$2 \quad T$-Selection Functions}

In the following two sections we assume that $T$ is a strong monad, and that $R$ is a $T$-algebra.

Definition 2.1 ( $T$-selection functions) Let $J_{R}^{T} X=(X \rightarrow R) \rightarrow T X$, where $R$ is a T-algebra. The elements of the type $J_{R}^{T} X$ will be called $T$-selection functions.

Under the assumptions that $T$ is a strong monad and $R$ a $T$-algebra, it follows that $J_{R}^{T}$ is also a strong monad.

Lemma 2.2 $J_{R}^{T}$ is a strong monad with operations:

(i) $\quad \eta_{X}^{J_{R}^{T}}(x)=\lambda p \cdot \eta_{X}^{T}(x)$

(ii) $\delta^{\dagger}(\varepsilon)=\lambda p \cdot\left(b_{p}^{\delta}\right)^{\dagger}\left(a_{p}^{\varepsilon, \delta}\right)$, where $\delta: X \rightarrow J_{R}^{T} Y$ and $\delta^{\dagger}: J_{R}^{T} X \rightarrow J_{R}^{T} Y$

where $b_{p}^{\delta}(x) \stackrel{T Y}{=} \delta(x)(p)$ and $a_{p}^{\varepsilon, \delta} \stackrel{T X}{=} \varepsilon\left(p^{*} \circ b_{p}^{\delta}\right)$. 
Proof. It is easy to check conditions $(i)$ and $(i i)$. Define $\Delta_{x}(p)=\left(b_{p}^{\delta}\right)^{\dagger}\left(a_{p}^{\varepsilon_{x}, \delta}\right)$ and $\Gamma_{\nu}(p)=\left(b_{p}^{\varepsilon}\right)^{\dagger}\left(a_{p}^{\nu, \varepsilon}\right)$. We outline property $(i i i)$ :

$$
\begin{array}{rll}
\left(\delta^{\dagger} \circ \varepsilon\right)^{\dagger} & = & \left(\lambda x \cdot \delta^{\dagger}\left(\varepsilon_{x}\right)\right)^{\dagger} \\
\stackrel{(i i)}{=} & \left(\lambda x \cdot \lambda p \cdot\left(b_{p}^{\delta}\right)^{\dagger}\left(a_{p}^{\varepsilon_{x}, \delta}\right)\right)^{\dagger} \\
\Delta \stackrel{\text { def. }}{=} & \left(\lambda x \cdot \Delta_{x}\right)^{\dagger} \\
\stackrel{(i i)}{=} & \lambda \nu \cdot \lambda q \cdot\left(b_{q}^{\Delta}\right)^{\dagger}\left(a_{q}^{\nu, \Delta}\right) \\
b \text { def. } & \lambda \nu \cdot \lambda q \cdot\left(\lambda x \cdot \Delta_{x}(q)\right)^{\dagger}\left(a_{q}^{\nu, \Delta}\right) \\
\stackrel{\text { def. }}{=} & \lambda \nu \cdot \lambda q \cdot\left(\left(b_{q}^{\delta}\right)^{\dagger} \circ\left(\lambda x \cdot a_{q}^{\varepsilon_{x}, \delta}\right)\right)^{\dagger}\left(a_{q}^{\nu, \Delta}\right) \\
\text { D[1.2 } & \text { iii) } & \lambda \nu \cdot \lambda q \cdot\left(\left(b_{q}^{\delta}\right)^{\dagger} \circ\left(\lambda x \cdot a_{q}^{\varepsilon_{x}, \delta}\right)^{\dagger}\right)\left(a_{q}^{\nu, \Delta}\right) \\
= & \lambda \nu \cdot \lambda q \cdot\left(b_{q}^{\delta}\right)^{\dagger}\left(\left(\lambda x \cdot a_{q}^{\varepsilon_{x}, \delta}\right)^{\dagger}\left(a_{q}^{\nu, \Delta}\right)\right) \\
& \stackrel{(*)}{=} & \lambda \nu \cdot \lambda q \cdot\left(b_{q}^{\delta}\right)^{\dagger}\left(a_{q}^{\Gamma_{\nu}, \delta}\right) \\
& \stackrel{(i i)}{=} & \lambda \nu \cdot \delta^{\dagger}\left(\Gamma_{\nu}\right) \\
\Gamma \stackrel{\text { def. }}{=} & \lambda \nu \cdot \delta^{\dagger}\left(\lambda p \cdot\left(b_{p}^{\varepsilon}\right)^{\dagger}\left(a_{p}^{\nu, \varepsilon}\right)\right) \\
\stackrel{(i i)}{=} & \lambda \nu \cdot \delta^{\dagger}\left(\varepsilon^{\dagger}(\nu)\right) \\
= & \delta^{\dagger} \circ \varepsilon^{\dagger} .
\end{array}
$$

It remains to show that $(*) a_{q}^{\Gamma_{\nu}, \delta}=\left(\lambda x \cdot a_{q}^{\varepsilon_{x}, \delta}\right)^{\dagger}\left(a_{q}^{\nu, \Delta}\right)$. This can be shown as

$$
\begin{array}{rrl}
a_{q}^{\Gamma_{\nu}, \delta} & a \text { def. } & \Gamma_{\nu}\left(q^{*} \circ b_{q}^{\delta}\right) \\
\Gamma \stackrel{\text { def. }}{=} & \left(\lambda x \cdot b_{q^{*} \circ b_{q}^{\delta}}^{\varepsilon}(x)\right)^{\dagger}\left(a_{q^{*} \circ b_{q}^{\delta}}^{\nu, \varepsilon}\right) \\
& \stackrel{\text { def. }}{=} & \left(\lambda x \cdot \varepsilon_{x}\left(q^{*} \circ b_{q}^{\delta}\right)\right)^{\dagger}\left(a_{q^{*} \circ b_{q}^{\delta}}^{\nu,}\right) \\
& \stackrel{(* *)}{=} & \left(\lambda x \cdot \varepsilon_{x}\left(q^{*} \circ b_{q}^{\delta}\right)\right)^{\dagger}\left(a_{q}^{\nu, \Delta}\right) \\
a \stackrel{\text { def. }}{=} & \left(\lambda x \cdot a_{q}^{\varepsilon_{x}, \delta}\right)^{\dagger}\left(a_{q}^{\nu, \Delta}\right),
\end{array}
$$

where, finally, $(* *) a_{q^{*} \circ b_{q}^{\delta}}^{\nu, \varepsilon}=a_{q}^{\nu, \Delta}$ is shown as

$$
\begin{array}{ccl}
a_{q^{*} \circ b_{q}^{\delta}}^{\nu, \varepsilon} & a \text { def. } & \nu\left(\left(q^{*} \circ b_{q}^{\delta}\right)^{*} \circ b_{q^{*} \circ b_{q}^{\delta}}^{\varepsilon}\right) \\
& { }^{\mathrm{D}[.3}(i i) & \nu\left(q^{*} \circ\left(b_{q}^{\delta}\right)^{\dagger} \circ b_{q^{*} \circ b_{q}^{\delta}}^{\varepsilon}\right) \\
= & \nu\left(\lambda x \cdot q^{*}\left(\left(b_{q}^{\delta}\right)^{\dagger}\left(b_{q^{*} \circ b_{q}^{\delta}}^{\varepsilon}(x)\right)\right)\right) \\
& b \text { def. } & \nu\left(\lambda x \cdot q^{*}\left(\left(b_{q}^{\delta}\right)^{\dagger}\left(\varepsilon_{x}\left(q^{*} \circ b_{q}^{\delta}\right)\right)\right)\right) \\
a \text { def. } & \nu\left(\lambda x \cdot q^{*}\left(\left(b_{q}^{\delta}\right)^{\dagger}\left(a_{q}^{\varepsilon_{x}, \delta}\right)\right)\right) \\
& \stackrel{\text { def. }}{=} & \nu\left(\lambda x \cdot q^{*}\left(\Delta_{x}(q)\right)\right) \\
b \text { def. } & \nu\left(\lambda x \cdot q^{*}\left(b_{q}^{\Delta}(x)\right)\right) \\
a \text { def. } & a_{q}^{\nu, \Delta .}
\end{array}
$$


It follows that the product operation of the monad $J_{R}^{T}$ can be explicitly described in terms of the product operation on $T$ as:

$$
\left(\varepsilon \otimes^{J_{R}^{T}} \delta\right)(q)=a \otimes^{T} f
$$

where $q: X \times Y \rightarrow R, \varepsilon:(X \rightarrow R) \rightarrow T X$ and $\delta: X \rightarrow(Y \rightarrow R) \rightarrow T Y$, and

$$
\begin{array}{lll}
f(x) & \stackrel{T Y}{=} & \delta_{x}\left(q_{x}\right) \\
a & \stackrel{T X}{=} & \varepsilon\left(\lambda x^{X} \cdot\left(q_{x}\right)^{*}(f x)\right) .
\end{array}
$$

Note that $\otimes$ on the right side of (2) denotes the product on the strong monad $T$ whereas $\otimes$ on the left denotes the product of the strong monad $J_{R}^{T}$. We will in general use the same notation $\otimes$ for the product of any strong monad, as it will hopefully be clear from the context which monad we are referring to.

Definition 2.3 (from $J_{R}^{T}$ to $\left.K_{R}\right)$ Let $K_{R} X=(X \rightarrow R) \rightarrow R$. Given a $T$ selection function $\varepsilon: J_{R}^{T} X$ we can construct a quantifier $\bar{\varepsilon}: K_{R} X$ as

$$
\bar{\varepsilon}\left(p^{X \rightarrow R}\right) \stackrel{R}{=} p^{*}(\varepsilon p) .
$$

It can be shown that the construction $\varepsilon \mapsto \bar{\varepsilon}$ is actually a monad morphism, from which the next lemma follows. Nevertheless, we shall prove the lemma directly. A particular instance of this lemma, when $T$ is the identity monad, was first proven in [5]. It is important here that $R$ is a $T$-algebra.

Lemma 2.4 Given $\varepsilon: J_{R}^{T} X$ and $\delta: X \rightarrow J_{R}^{T} Y$ then

$$
\overline{\left(\varepsilon \otimes J_{R}^{T} \delta\right)}=\bar{\varepsilon} \otimes^{K_{R}}\left(\lambda x \cdot \overline{\delta_{x}}\right) .
$$

Proof. Define $f(x)=\delta_{x}\left(q_{x}\right)$ and $p(x)=\left(q_{x}\right)^{*}(f x)$ and $a=\varepsilon(p)$. We calculate as follows:

$$
\begin{aligned}
& \overline{\left(\varepsilon \otimes^{J_{R}^{T}} \delta\right)}(q) \quad \mathrm{D} \underline{\underline{\underline{2.3}}} \quad q^{*}\left(\left(\varepsilon \otimes^{J_{R}^{T}} \delta\right)(q)\right) \\
& \stackrel{2}{\underline{\underline{2}}} \quad q^{*}\left(a \otimes^{T} f\right) \\
& \text { L1.4 }\left(\lambda x \cdot\left(q_{x}\right)^{*}(f x)\right)^{*}(a) \\
& \stackrel{\operatorname{Def}(a)}{=} \quad\left(\lambda x \cdot\left(q_{x}\right)^{*}(f x)\right)^{*}(\varepsilon(p)) \\
& \stackrel{\operatorname{Def}(p)}{=} \quad p^{*}(\varepsilon(p)) \\
& \mathrm{D} \underline{\underline{2.3}} \bar{\varepsilon}(p) \\
& \operatorname{Def} \underline{\underline{p}}, f) \quad \bar{\varepsilon}\left(\lambda x \cdot\left(q_{x}\right)^{*}\left(\delta_{x}\left(q_{x}\right)\right)\right) \\
& \mathrm{D} \underline{\underline{\underline{2.3}}} \bar{\varepsilon}\left(\lambda x \cdot \overline{\delta_{x}}\left(q_{x}\right)\right) \\
& =\left(\bar{\varepsilon} \otimes K_{R}\left(\lambda x . \overline{\delta_{x}}\right)\right)(q) .
\end{aligned}
$$

The last equality in the chain above uses the definition of the product $\otimes$ for the strong monad $K_{R} X$. 


\section{Iterated Products and Bar Recursion}

Given any strong monad $M$ we can iterate its product operation $M X \times(X \rightarrow$ $M Y) \rightarrow M(X \times Y)$ so as to obtain an operation 2 on infinite sequences $\left(X^{*} \rightarrow\right.$ $M(X))^{\mathbb{N}} \rightarrow M\left(X^{\mathbb{N}}\right)$. Although this will not be a total operation in general, it is surprising that, as shown in [5], it defines a total operation when $M$ is the selection monad $M X=J_{R} X$ and $R$ is a discrete type.

It is also possible to iterate the binary product of $M$ in a controlled way, by using an explicit termination function $\omega: X^{\mathbb{N}} \rightarrow \mathbb{N}$ as

$$
M-\mathrm{EP}_{s}^{\omega}(\alpha)= \begin{cases}\eta^{M}(\langle\rangle) & \text { if } \omega\left(s^{+}\right)<|s| \\ \alpha_{s} \otimes^{M}\left(\lambda x \cdot M-\mathrm{EP}_{s * x}^{\omega}(\alpha)\right. & \text { otherwise }\end{cases}
$$

where $M$-EP ${ }_{s}^{\omega}$ is of type $\left(\mathbb{N} \rightarrow M(X) \rightarrow M\left(X^{*}\right)\right.$. We use the acronym $M$-EP for the "explicitly controlled iterated product of the strong monad $M$ ".

The explicitly controlled product of selection functions EPS or quantifiers EPQ (cf. [7]) are particular cases when $M X=J_{R} X$ and $M X=K_{R} X$, this time for an arbitrary $R$, i.e. EPS $=J_{R}$-EP and EPQ $=K_{R}$-EP. In turn, these are primitively recursively equivalent to restricted Spector bar recursion and the general Spector bar recursion, respectively [7].

In this section we consider another instance where $M X=J_{R}^{T} X$, with $T$ being a strong monad, i.e. $J_{R}^{T}$-EP which we shall call $T$-EPS.

Definition 3.1 (Iterated $J_{R}^{T}$ product) Let $\varepsilon_{s}: J_{R}^{T} X_{|s|}$ and $s: X^{*}$ and $\omega: X^{\mathbb{N}} \rightarrow$ $\mathbb{N}$. We define $T$-EPS ${ }_{s}^{\omega}(\varepsilon): J_{R}^{T} X^{*}$ as $T$-EPS ${ }_{s}^{\omega}=J_{R}^{T}$-EP ${ }_{s}^{\omega}$.

Unfolding the definition of the binary product, as in Lemma 2.2, and noticing that $\eta^{J_{R}^{T}}(\langle\rangle)=\lambda q \cdot \eta^{T}(\langle\rangle)$, the equation above can be also written as

$$
T-\operatorname{EPS}_{s}^{\omega}(\varepsilon)(q)= \begin{cases}\eta^{T}(\langle\rangle) & \text { if } \omega\left(s^{+}\right)<|s| \\ a \otimes^{T} f & \text { otherwise }\end{cases}
$$

where $a=\varepsilon_{s}\left(\lambda x \cdot\left(q_{x}\right)^{*}(f x)\right)$ and $f(x)=T$-EPS ${ }_{s * x}^{\omega}(\varepsilon)\left(q_{x}\right)$.

Recall that EPQ is the explicitly controlled iterated product of quantifiers, i.e. $E P Q=K_{R}$-EP. EPQ satisfies the equation

$$
\mathrm{EPQ}_{s}^{\omega}(\phi)= \begin{cases}\lambda q \cdot q(\langle\rangle) & \text { if } \omega\left(s^{+}\right)<|s| \\ \phi_{s} \otimes^{K_{R}} \lambda x . \mathrm{EPQ}_{s * x}^{\omega}(\phi) & \text { otherwise. }\end{cases}
$$

Again, the definition of the binary product of quantifiers can unfolded, leading to the equivalent equation

$$
\mathrm{EPQ}_{s}^{\omega}(\phi)(q)= \begin{cases}q(\langle\rangle) & \text { if } \omega\left(s^{+}\right)<|s| \\ \phi_{s}\left(\lambda x . \mathrm{EPQ}_{s * x}^{\omega}(\phi)\left(q_{x}\right)\right) & \text { otherwise }\end{cases}
$$

\footnotetext{
${ }^{2} \mathrm{~A}$ simpler instance of this operation without dependent types, namely $(M X)^{\mathbb{N}} \rightarrow M\left(X^{\mathbb{N}}\right)$, is actually a built-in function in standard implementations of the Haskell programming language called sequence :: Monad $\mathrm{m}=>[\mathrm{m}$ a] $->\mathrm{m}$ [a].
} 
As show in [4], EPQ is equivalent over system $T$ to Spector's bar recursion. The following lemma follows by a simple iteration of Lemma 2.4 .

Lemma 3.2 $\overline{T-\operatorname{EPS}_{\langle\rangle}^{\omega}(\varepsilon)}=\mathrm{EPQ}_{\langle\rangle}^{\omega}(\bar{\varepsilon})$.

Proof. The proof goes by bar induction on $s$ with the bar $\omega\left(s^{+}\right)<|s|$. In case we have reached the bar, i.e. $\omega\left(s^{+}\right)<|s|$, we have

$$
\begin{array}{ccl}
\mathrm{EPQ}_{s}^{\omega}(\bar{\varepsilon})(q) & = & q(\langle\rangle) \\
& \underline{\mathrm{D} \underline{\underline{1.3}}} i) & q^{*}\left(\eta^{T}(\langle\rangle)\right) \\
\mathrm{D} \underline{\underline{\underline{3.1}}} & q^{*}\left(T-\operatorname{EPS}_{s}^{\omega}(\varepsilon)(q)\right) \\
\mathrm{D} \underline{\underline{\underline{2.3}}} & \overline{T-\operatorname{EPS}_{s}^{\omega}(\varepsilon) .}
\end{array}
$$

By the bar inductive assumption we have that $\overline{T-\mathrm{EPS}_{s * x}^{\omega}(\varepsilon)}=\mathrm{EPQ}_{s * x}^{\omega}(\bar{\varepsilon})$, for all $x$, and hence

$$
\begin{aligned}
& \mathrm{EPQ}_{s}^{\omega}(\bar{\varepsilon})(q)=\left(\bar{\varepsilon} \otimes^{K_{R}}\left(\lambda x . \mathrm{EPQ}_{s * x}^{\omega}(\bar{\varepsilon})\right)\right)(q) \\
& \stackrel{(\mathrm{IH})}{=}\left(\bar{\varepsilon} \otimes K^{K_{R}}\left(\lambda x \cdot \overline{T-\mathrm{EPS}_{s * x}^{\omega}(\varepsilon)}\right)\right)(q) \\
& \left.\mathrm{L} \underline{\underline{\underline{2.4}}} \overline{\left(\varepsilon \otimes^{J_{R}^{T}}\left(\lambda x \cdot T-\mathrm{EPS}_{s * x}^{\omega}(\varepsilon)\right)\right.}\right)(q) \\
& =\overline{T-\operatorname{EPS}_{s}^{\omega}(\varepsilon)}(q),
\end{aligned}
$$

since we can assume $\omega\left(s^{+}\right) \geq|s|$.

It is well know that the product of selection functions of type $(X, R)$ can be simulated by a product where $R$ is restricted to $R=X^{\mathbb{N}}$ and $q: X^{\mathbb{N}} \rightarrow R$ is the identity function. In fact, one can think of Spector's restricted form of bar recursion [16] as the iterated product of these restricted selection functions. In terms of games, it corresponds to taking the outcome of the game to be the sequence of moves played. The actual outcome of the game can be reconstructed from this sequence via the outcome function. The next lemma shows that this simulation of an arbitrary outcome type $R$ by taking the outcome to be the actual sequence of moves also works in this monadic setting.

Lemma 3.3 T-EPS of type $(X, R)$ is definable from $T$-EPS of type $\left(X, T X^{\mathbb{N}}\right)$.

Proof. Let $\operatorname{add}_{s}: X^{\mathbb{N}} \rightarrow X^{\mathbb{N}}$ and $\operatorname{drop}_{n}: X^{\mathbb{N}} \rightarrow X^{\mathbb{N}}$ be the functions that append the finite sequence $s$ to the beginning of an infinite list, and the function that drops $n$ elements from an infinite list, respectively. Clearly, $\operatorname{drop}_{|s|} \circ \operatorname{add}_{s}$ is the identity, and hence, by functoriality, $T\left(\operatorname{drop}_{|s|}\right) \circ T\left(\operatorname{add}_{s}\right)$ is the identity on $T\left(X^{\mathbb{N}}\right)$. Given $q: X^{\mathbb{N}} \rightarrow R$ and $\varepsilon_{s}: J_{R}^{T} X$ we define $\varepsilon_{s}^{q}: J_{T X^{\mathbb{N}}}^{T} X$ as

$$
\varepsilon_{s}^{q}\left(p^{X \rightarrow T X^{\mathbb{N}}}\right) \stackrel{T X}{=} \varepsilon_{s}\left(\lambda x \cdot\left(\left(q_{s * x}\right)^{*} \circ T\left(\operatorname{drop}_{|s * x|}\right)\right)(p x)\right) .
$$


Note that $T X^{\mathbb{N}}$ is also a $T$-algebra with the map

$$
(\cdot)^{*}:\left(Y \rightarrow T X^{\mathbb{N}}\right) \rightarrow\left(T Y \rightarrow T X^{\mathbb{N}}\right)
$$

being simply the $(\cdot)^{\dagger}$ of the monad $T$. We claim that

$$
T-\operatorname{EPS}_{\langle\rangle}^{\omega}(\varepsilon)(q)=T-\operatorname{EPS}_{\langle\rangle}^{\omega}\left(\varepsilon^{q}\right)\left(\eta^{T}\right) .
$$

Define

$$
P(s) \equiv T-\operatorname{EPS}_{s}^{\omega}(\varepsilon)\left(q_{s}\right)=T-\operatorname{EPS}_{s}^{\omega}\left(\varepsilon^{q}\right)\left(\eta^{T} \circ \operatorname{add}_{s}\right)
$$

and let us show $P(\langle\rangle)$ by bar induction. Recall that $T\left(\operatorname{add}_{s}\right)=\eta^{T} \circ \operatorname{add}_{s}$ by definition. In the base case, assuming $\omega\left(s^{+}\right)<|s|$, we have

$$
T-\operatorname{EPS}_{s}^{\omega}(\varepsilon)\left(q_{s}\right)=\eta^{T}(\langle\rangle)=T-\operatorname{EPS}_{s}^{\omega}\left(\varepsilon^{q}\right)\left(\eta^{T} \circ \operatorname{add}_{s}\right) .
$$

For the bar inductive step we assume $P(s * x)$ holds for all $x$ and must prove $P(s)$. We can also assume that $\omega\left(s^{+}\right) \geq|s|$. Let

$$
\begin{aligned}
f(x) & =T-\operatorname{EPS}_{s * x}^{\omega}(\varepsilon)\left(q_{s * x}\right) \\
a & =\varepsilon_{s}\left(\lambda x \cdot\left(q_{s * x}\right)^{*}(f x)\right) \\
\tilde{f}(x) & =T-\operatorname{EPS}_{s * x}^{\omega}\left(\varepsilon^{q}\right)\left(\eta^{T} \circ \operatorname{add}_{s * x}\right) \\
\tilde{a} & =\varepsilon_{s}^{q}\left(\lambda x \cdot T\left(\operatorname{add}_{s * x}\right)(\tilde{f} x)\right) .
\end{aligned}
$$

By the bar inductive hypothesis we have $f=\tilde{f}$ and hence

$$
\begin{array}{rll}
\tilde{a} & \stackrel{=}{(\mathrm{IH})} & \varepsilon_{s}^{q}\left(\lambda x \cdot T\left(\operatorname{add}_{s * x}\right)(\tilde{f} x)\right) \\
& \stackrel{(\mathrm{IH}}{=} & \varepsilon_{s}^{q}\left(\lambda x \cdot T\left(\operatorname{add}_{s * x}\right)(f x)\right) \\
& \stackrel{\left(\varepsilon^{q} \text { def }\right)}{=} & \left.\varepsilon_{s}\left(\lambda x \cdot\left(\left(q_{s * x}\right)^{*} \circ T\left(\operatorname{drop}_{|s * x|}\right)\right)\left(T\left(\operatorname{add}_{s * x}\right)(f x)\right)\right)\right) \\
& = & \varepsilon_{s}\left(\lambda x \cdot\left(q_{s * x}\right)^{*}(f x)\right) \\
& = & a .
\end{array}
$$

Therefore

$$
\begin{aligned}
T-\operatorname{EPS}_{s}^{\omega}(\varepsilon)\left(q_{s}\right) & =a \otimes^{T} f \\
& =\tilde{a} \otimes^{T} \tilde{f} \\
& =T-\operatorname{EPS}_{s}^{\omega}\left(\varepsilon^{q}\right)\left(\eta^{T} \circ \operatorname{add}_{s}\right) .
\end{aligned}
$$

In the last step we have used that $T\left(\operatorname{add}_{s}\right)$ is defined as $\eta^{T} \circ \operatorname{add}_{s}$.

The main result in this section is that Spector's original bar recursion already defines the explicitly controlled product of $T$-selection functions $T$-EPS. Spector proves this in [16] for the case when $T$ is the identity monad. The following theorem shows that this in fact holds for any strong monad $T$.

Theorem 3.4 T-EPS is definable from EPQ. 
Proof. We claim that $T$-EPS $\operatorname{ES}_{\langle\rangle}^{\omega}(\varepsilon)(q)$ can be defined as $\mathrm{EPQ}_{\langle\rangle}^{\omega}\left(\overline{\varepsilon^{q}}\right)(\eta)$, where $\varepsilon^{q}$ is as in the proof of the previous lemma. Indeed we have:

$$
\begin{array}{rll}
\mathrm{EPQ}_{\langle\rangle}^{\omega}\left(\overline{\varepsilon^{q}}\right)(\eta) & \mathrm{L} \underline{\underline{\underline{3.2}}} & \overline{T-\mathrm{EPS}_{\langle\rangle}^{\omega}\left(\varepsilon^{q}\right)}(\eta) \\
\mathrm{D} \underline{\underline{\underline{2.3}}} & \eta^{*}\left(T-\mathrm{EPS}_{\langle\rangle}^{\omega}\left(\varepsilon^{q}\right)(\eta)\right) \\
\mathrm{L} \underline{\underline{3.3}} & \eta^{*}\left(T-\operatorname{EPS}_{\langle\rangle}^{\omega}(\varepsilon)(q)\right) \\
= & \eta^{\dagger}\left(T-\operatorname{EPS}_{\langle\rangle}^{\omega}(\varepsilon)(q)\right) \\
\mathrm{D} \underline{\underline{\underline{1.2}}} i) & T-\operatorname{EPS}_{\langle\rangle}^{\omega}(\varepsilon)(q) .
\end{array}
$$

We used that the map $(\cdot)^{*}$ for the algebra $T X^{\mathbb{N}}$ is just the $(\cdot)^{\dagger}$ map for the monad $T$, as discussed in the proof of Lemma 3.3 .

\section{Finite Power Sets}

For the rest of the paper we will make essential use of the definitional extension of Gödel's system $\mathrm{T}$ with the finite power-set type $\mathcal{P}_{\mathrm{f}}(X)$. To simplify the exposition, let us also abbreviate $\mathcal{P}_{\mathrm{f}}(X \rightarrow Y)$ as $X \Rightarrow Y$, i.e. the type of finite sets of functions from $X$ to $Y$. We can think of the elements $f: X \Rightarrow \mathcal{P}_{\mathrm{f}}(Y)$ as functions by defining the following set-application

$$
\operatorname{Ap}(f)\left(x^{X}\right) \stackrel{\mathcal{P}_{\mathrm{f}}(Y)}{=} \bigcup_{g \in f} g x
$$

Hence, if $f: X \Rightarrow \mathcal{P}_{\mathrm{f}}(Y)$ then $\operatorname{Ap}(f)(\cdot): X \rightarrow \mathcal{P}_{\mathrm{f}}(Y)$. In particular, if $f:(X \Rightarrow(Y \Rightarrow$ $\left.\left.\mathcal{P}_{\mathrm{f}}(Z)\right)\right)$ then $\operatorname{Ap}(\operatorname{Ap}(f)(x))(y)$ stands for

$$
\bigcup_{g \in f} \bigcup_{h \in g x} h y
$$

and we will be abbreviated that as $\operatorname{Ap}^{2}(f)(x, y)$.

Lemma 4.1 The finite power set type operator $\mathcal{P}_{\mathrm{f}}(\cdot)$ is a strong monad with operations

- $\eta(x)=\{x\}$

- $f^{\dagger}(S)=\bigcup\{f(x): x \in S\}$, for $f: X \rightarrow \mathcal{P}_{\mathrm{f}}(Y)$.

Moreover, its binary product

$$
\otimes: \mathcal{P}_{\mathrm{f}}(X) \times\left(X \rightarrow \mathcal{P}_{\mathrm{f}}(Y)\right) \rightarrow \mathcal{P}_{\mathrm{f}}(X \times Y)
$$

can be explicitly described as

$$
S \otimes f=\{\langle a, b\rangle: a \in S \wedge b \in f(a)\} .
$$


For the rest of the paper we shall assume that $R=\mathcal{P}_{\mathrm{f}}\left(R^{\prime}\right)$, for some $R^{\prime}$, so that $R$ is an algebra for $\mathcal{P}_{\mathrm{f}}(\cdot)$ with $(\cdot)^{*}=(\cdot)^{\dagger}$. We will also use $\bigcup: \mathcal{P}_{\mathrm{f}}(R) \rightarrow R$, the usual union operation which satisfies $S_{i} \subseteq \bigcup\left\{S_{i}: i \in I\right\}$ (we use this in Lemma 4.6).

Definition 4.2 (Herbrand bar recursion) Let us write hBR for the instance of $T$-EPS where $T=\mathcal{P}_{\mathrm{f}}(\cdot)$, i.e

$$
\operatorname{hBR}_{s}^{\omega}(\varepsilon)(q)= \begin{cases}\{\langle\rangle\} & \text { if } \omega\left(s^{+}\right)<|s| \\ \left\{a * r: a \in \chi \wedge r \in \operatorname{hBR}_{s * a}(\omega)(\varepsilon)\left(q_{a}\right)\right\} & \text { otherwise }\end{cases}
$$

where $\chi=\varepsilon_{s}\left(\lambda x . \bigcup\left\{q_{x}(r): r \in \mathrm{hBR}_{s * x}(\omega)(\varepsilon)\left(q_{x}\right)\right\}\right)$.

By Theorem 3.4hBR is T-definable from Spector's general form of bar recursion 14]. We now prove four lemmas about $h B R$, to be used in the interpretation of DNS in the following section. For this section we will assume that $\varepsilon$ and $\omega$ are fixed functionals and hence, for the sake of readability, we shall omit these as parameters in $\operatorname{hBR}_{s}^{\omega}(\varepsilon)(q)$.

Lemma 4.3 Let $t=\mathrm{hBR}_{\langle\rangle}(q)$ and $s \in t$. For all $i \leq|s|$ we have

$$
s \in\left\{\left\langle s_{0}, \ldots, s_{i-1}\right\rangle * r: r \in \mathrm{hBR}_{\left\langle s_{0}, \ldots, s_{i-1}\right\rangle}\left(q_{\left\langle s_{0}, \ldots, s_{i-1}\right\rangle}\right)\right\} .
$$

The types are $t: \mathcal{P}_{\mathrm{f}}\left(X^{*}\right)$ and $s: X^{*}$.

Proof. By induction on $i$. If $i=0$ then $\left\langle s_{0}, \ldots, s_{i-1}\right\rangle$ is the empty sequence and the result follows by the assumption that $s \in t$. For the induction step assume that $i<|s|$ and that

$$
s \in\left\{\left\langle s_{0}, \ldots, s_{i-1}\right\rangle * r: r \in \mathrm{hBR}_{\left\langle s_{0}, \ldots, s_{i-1}\right\rangle}\left(q_{\left\langle s_{0}, \ldots, s_{i-1}\right\rangle}\right\rangle\right) .
$$

Since $i<|s|$ there must exist some $r \in \mathrm{hBR}_{\left\langle s_{0}, \ldots, s_{i-1}\right\rangle}\left(q_{\left\langle s_{0}, \ldots, s_{i-1}\right\rangle}\right)$ of the form $s_{i} * r^{\prime}$ so that

(i) $s=\left\langle s_{0}, \ldots, s_{i-1}, s_{i}\right\rangle * r^{\prime}$, and

(ii) $s_{i} * r^{\prime} \in \mathrm{hBR}_{\left\langle s_{0}, \ldots, s_{i-1}\right\rangle}\left(q_{\left\langle s_{0}, \ldots, s_{i-1}\right\rangle}\right)$.

In particular, we cannot have $\mathrm{hBR}_{\left\langle s_{0}, \ldots, s_{i-1}\right\rangle}\left(q_{\left\langle s_{0}, \ldots, s_{i-1}\right\rangle}\right)=\{\langle\rangle\}$, so it must be the case that $(*) \omega\left(\left\langle s_{0}, \ldots, s_{i-1}\right\rangle^{+}\right) \geq\left|\left\langle s_{0}, \ldots, s_{i-1}\right\rangle\right|$. Hence

$$
\operatorname{hBR}_{\left\langle s_{0}, \ldots, s_{i-1}\right\rangle}\left(q_{\left\langle s_{0}, \ldots, s_{i-1}\right\rangle}\right)=\left\{a * r: a \in \chi \wedge r \in \operatorname{hBR}_{\left\langle s_{0}, \ldots, s_{i-1}, a\right\rangle}\left(q_{\left\langle s_{0}, \ldots, s_{i-1}, a\right\rangle}\right)\right\}
$$

where

$$
\chi=\varepsilon_{\left\langle s_{0}, \ldots, s_{i-1}\right\rangle}\left(\lambda y^{X} \cdot \bigcup\left\{q_{\left\langle s_{0}, \ldots, s_{i-1}, y\right\rangle}(r): r \in \mathrm{hBR}_{\left\langle s_{0}, \ldots, s_{i-1}, y\right\rangle}\left(q_{\left\langle s_{0}, \ldots, s_{i-1}, y\right\rangle}\right)\right\}\right) .
$$

From (ii) it follows that $s_{i} \in \chi$ and

(iii) $r^{\prime} \in \operatorname{hBR}_{\left\langle s_{0}, \ldots, s_{i-1}, s_{i}\right\rangle}\left(q_{\left\langle s_{0}, \ldots, s_{i-1}, s_{i}\right\rangle}\right)$. 
Finally, from (i) and (iii) we have

$$
s \in\left\{\left\langle s_{0}, \ldots, s_{i-1}, s_{i}\right\rangle * r^{\prime}: r^{\prime} \in \mathrm{hBR}_{\left\langle s_{0}, \ldots, s_{i-1}, s_{i}\right\rangle}\left(q_{\left\langle s_{0}, \ldots, s_{i-1}, s_{i}\right\rangle}\right)\right\}
$$

which concludes the proof.

For the following three lemmas let $t=\mathrm{hBR}_{\langle\rangle}(q)$, and assume $a_{0}, \ldots, a_{n}$ is a finite sequence satisfying, for all $i \leq n$,

$$
a_{i} \in \varepsilon_{\left\langle a_{0}, \ldots, a_{i-1}\right\rangle}\left(p_{i}\right)
$$

where $p_{i}$ is defined as

$$
p_{i}(y)=\bigcup\left\{q_{\left\langle a_{0}, \ldots, a_{i-1}, y\right\rangle}(r): r \in \operatorname{hBR}_{\left\langle a_{0}, \ldots, a_{i-1}, y\right\rangle}\left(q_{\left\langle a_{0}, \ldots, a_{i-1}, y\right\rangle}\right)\right\} .
$$

Lemma 4.4 If $\omega\left(\left\langle a_{0}, \ldots, a_{i-1}\right\rangle^{+}\right) \geq i$, for all $i \leq n$, then

$$
\left\langle a_{0}, \ldots, a_{n-1}\right\rangle * x * r \in t,
$$

for all $x \in \varepsilon_{\left\langle a_{0}, \ldots, a_{n-1}\right\rangle}\left(p_{n}\right)$ and $r \in \mathrm{hBR}_{\left\langle a_{0}, \ldots, a_{n-1}, x\right\rangle}\left(q_{\left\langle a_{0}, \ldots, a_{n-1}, x\right\rangle}\right)$.

Proof. We prove the lemma by induction on $n$.

For $n=0$ the assumption of the lemma always holds, while the conclusion follows by the definition of $h B R$

$$
t=\operatorname{hBR}_{\langle\rangle}(q)=\left\{a * r: a \in \varepsilon_{\langle\rangle}\left(p_{0}\right) \wedge r \in \operatorname{hBR}_{a}\left(q_{a}\right)\right\}
$$

since $\omega\left(\langle\rangle^{+}\right) \geq 0$. For the induction step, assume that $\omega\left(\left\langle a_{0}, \ldots, a_{i-1}\right\rangle^{+}\right) \geq i$, for all $i \leq n+1$. In particular this holds for $i \leq n$. Hence, by induction hypothesis we have

$$
\left\langle a_{0}, \ldots, a_{n-1}\right\rangle * x * r \in t
$$

$$
\text { for all } x \in \varepsilon_{\left\langle a_{0}, \ldots, a_{n-1}\right\rangle}\left(p_{n}\right) \text { and } r \in \mathrm{hBR}_{\left\langle a_{0}, \ldots, a_{n-1}, x\right\rangle}\left(q_{\left\langle a_{0}, \ldots, a_{n-1}, x\right\rangle}\right)
$$

and, since $a_{n} \in \varepsilon_{\left\langle a_{0}, \ldots, a_{n-1}\right\rangle}\left(p_{n}\right)$,

(i) $\left\langle a_{0}, \ldots, a_{n}\right\rangle * r \in t$, for all $r \in \mathrm{hBR}_{\left\langle a_{0}, \ldots, a_{n}\right\rangle}\left(q_{\left\langle a_{0}, \ldots, a_{n}\right\rangle}\right)$.

Now fix a $y \in \varepsilon_{\left\langle a_{0}, \ldots, a_{n}\right\rangle}\left(p_{n+1}\right)$ and an $r^{\prime} \in \operatorname{hBR}_{\left\langle a_{0}, \ldots, a_{n}, y\right\rangle}\left(q_{\left\langle a_{0}, \ldots, a_{n}, y\right\rangle}\right)$. In order to show that $\left\langle a_{0}, \ldots, a_{n}\right\rangle * y * r^{\prime} \in t$, by $(i)$ it is enough to show that $y * r^{\prime} \in$ $\operatorname{hBR}_{\left\langle a_{0}, \ldots, a_{n}\right\rangle}\left(q_{\left\langle a_{0}, \ldots, a_{n}\right\rangle}\right)$. But since $\omega\left(\left\langle a_{0}, \ldots, a_{n}\right\rangle^{+}\right) \geq n+1$, this indeed follows by the definition of $\mathrm{hBR}$, and the assumptions on $y$ and $r^{\prime}$.

Lemma 4.5 Let $t$ and $a_{i}$ 's be as above. Define $N=1+\max \{|s|: s \in t\}$. For some $n<N$ we have that

(a) $n$ is the least such that $\omega\left(\left\langle a_{0}, \ldots, a_{n}\right\rangle^{+}\right)<n+1$, and

(b) $\left\langle a_{0}, \ldots, a_{n}\right\rangle \in t$. 
Proof. Suppose that for all $n \leq N$ we have $\omega\left(\left\langle a_{0}, \ldots, a_{n-1}\right\rangle^{+}\right) \geq n$. By Lemma 4.4 this would imply $\left\langle a_{0}, \ldots, a_{N-1}\right\rangle * r \in t$ for some non-empty finite sequence $r$, which is a contradiction by the definition of $N$. Therefore, let $n<N$ be the smallest such that $\omega\left(\left\langle a_{0}, \ldots, a_{n}\right\rangle^{+}\right)<n+1$, so that for all $i \leq n$ we have $\omega\left(\left\langle a_{0}, \ldots, a_{i-1}\right\rangle^{+}\right) \geq i$. By Lemma 4.4 again we have that $\left\langle a_{0}, \ldots, a_{n-1}\right\rangle * a_{n} * r \in t$ for all $r \in \mathrm{hBR}_{\left\langle a_{0}, \ldots, a_{n}\right\rangle}\left(q_{\left\langle a_{0}, \ldots, a_{n}\right\rangle}\right)$. But since $\omega\left(\left\langle a_{0}, \ldots, a_{n}\right\rangle^{+}\right)<n+1$ we have that $\mathrm{hBR}_{\left\langle a_{0}, \ldots, a_{n}\right\rangle}\left(q_{\left\langle a_{0}, \ldots, a_{n}\right\rangle}\right)=\{\langle\rangle\}$, implying $\left\langle a_{0}, \ldots, a_{n}\right\rangle \in t$.

Lemma 4.6 Let $t, p_{i}, a_{i}$ be as above, and $n<N$ as in Lemma 4.5. Let also $s=\left\langle a_{0}, \ldots, a_{n}\right\rangle$. Then for all $i \leq n$

$$
q(s) \subseteq p_{i}\left(a_{i}\right) .
$$

Proof. By Lemma 4.5 we have that $s \in t$. Hence, by Lemma 4.3, for $i \leq n$

$$
s \in\left\{\left\langle a_{0}, \ldots, a_{i-1}, a_{i}\right\rangle * r: r \in \mathrm{hBR}_{\left\langle a_{0}, \ldots, a_{i-1}, a_{i}\right\rangle}\left(q_{\left\langle a_{0}, \ldots, a_{i-1}, a_{i}\right\rangle}\right)\right\} .
$$

It follows that

$$
q(s) \in\left\{q\left(\left\langle a_{0}, \ldots, a_{i-1}, a_{i}\right\rangle * r\right): r \in \mathrm{hBR}_{\left\langle a_{0}, \ldots, a_{i-1}, a_{i}\right\rangle}\left(q_{\left\langle a_{0}, \ldots, a_{i-1}, a_{i}\right\rangle}\right)\right\} .
$$

Hence

$$
\begin{aligned}
q(s) & \subseteq \bigcup\left\{q_{\left\langle a_{0}, \ldots, a_{i-1}, a_{i}\right\rangle}(r): r \in \mathrm{hBR}_{\left\langle a_{0}, \ldots, a_{i-1}, a_{i}\right\rangle}\left(q_{\left\langle a_{0}, \ldots, a_{i-1}, a_{i}\right\rangle}\right)\right\} \\
& =p_{i}\left(a_{i}\right)
\end{aligned}
$$

which concludes the proof.

\section{Application: Herbrand Interpretation of DNS}

In this final section we show how the product of $T$-selection functions, with $T$ being the finite power-set monad, witnesses the Herbrand functional interpretation of the double negation shift

$$
\text { DNS : } \quad \forall^{\text {st }} n^{\mathbb{N}} \neg \neg A(n) \rightarrow \neg \neg \forall^{\text {st }} n^{\mathbb{N}} A(n)
$$

where $\forall^{\text {st }} x A(x)$ is the quantification over standard objects from 18 . Let us first briefly recall here the definition of the Herbrand functional interpretation from 18 . We shall only present the $\left\{\rightarrow, \forall^{\text {st }}, \perp\right\}$-fragment as this is enough to carry out the interpretation of DNS. Negation $\neg A$ is defined as $A \rightarrow \perp$. Although we will present an explicit definition for the witnesses of DNS, for simplicity, we will carry out the verification of correctness in a classical setting, reading the weak existential $\neg \forall x \neg A$ as the strong one $\exists x A$.

Definition 5.1 ([18]) The Herbrand functional interpretation of a formula $A$ is defined by structural induction. Assum $\S^{3}(A)^{H}=\exists^{\text {st }} a^{X} \forall^{\text {st }} b^{Y} A_{H}(a, b)$ and $(B)^{H}=$

\footnotetext{
${ }^{3}$ Here $a, b, c$ and $d$ are potentially tuples of variables, though for simplicity we will treat them as if they were single variables.
} 
$\exists^{\text {st }} c^{V} \forall^{\text {st }} d^{W} B_{H}(c, d)$. The only relevant cases for the interpretation of DNS are:

$$
\begin{array}{ll}
(\perp)^{H} & \equiv \perp \\
(A \rightarrow B)^{H} & \equiv \exists^{\text {st }} f, g \forall^{\text {st }} a^{X}, d^{W}\left(\forall b \in \operatorname{Ap}^{2}(g)(a, d) A_{H}(a, b) \rightarrow B_{H}(\operatorname{Ap}(f)(a), d)\right) \\
\left(\forall^{\text {st }} z^{Z} A\right)^{H} & \equiv \exists^{\text {st }} h^{Z \Rightarrow X}{ }^{\text {st }} z, b A_{H}(\operatorname{Ap}(h)(z), b)
\end{array}
$$

where in the clause for $A \rightarrow B$ the types of $f$ and $g$ are

$$
\begin{aligned}
& f \quad: \quad X \Rightarrow V \\
& g \quad: \quad X \Rightarrow\left(W \Rightarrow \mathcal{P}_{\mathrm{f}}(Y)\right) .
\end{aligned}
$$

For all other cases, including the other base cases, see [18].

Let us start by working out the Herbrand interpretation of negation $\neg A$ and double-negation $\neg \neg A$. If $(A)^{H}=\exists^{\text {st }} a^{X} \forall^{\text {st }} b^{R} A_{H}(a, b)$ then

$$
(\neg A)^{H} \equiv \exists^{\mathrm{st}} p^{X \Rightarrow \mathcal{P}_{\mathrm{f}}(R)} \forall^{\mathrm{st}} a^{X} \neg \forall b \in \operatorname{Ap}(p)(a) A_{H}(a, b)
$$

and hence

$$
(\neg \neg A)^{H} \equiv \exists^{\text {st }} \varepsilon \forall^{\text {st }} p^{X \Rightarrow \mathcal{P}_{\mathrm{f}}(R)} \exists a^{X} \in \operatorname{Ap}(\varepsilon)(p) \forall b \in \operatorname{Ap}(p)(a) A_{H}(a, b)
$$

where $\varepsilon:\left(X \Rightarrow \mathcal{P}_{\mathrm{f}}(R)\right) \Rightarrow \mathcal{P}_{\mathrm{f}}(X)$. Assuming $A(n)$ has a Herbrand functional interpretation $\exists^{\text {st }} a^{X} \forall^{\text {st }} b^{R} A_{H}(n, a, b)$ then the interpretation of $\forall^{\text {st }} n^{\mathbb{N}} \neg \neg A(n)$ is

$$
\exists^{\text {st }} \delta \forall^{\text {st }} n \forall^{\text {st }} p^{X \Rightarrow \mathcal{P}_{\mathrm{f}}(R)} \exists a \in \operatorname{Ap}^{2}(\delta)(n, p) \forall b \in \operatorname{Ap}(p)(a) A_{H}(n, a, b) .
$$

The interpretation of the conclusion of DNS, $\neg \neg \forall^{\text {st }} n^{\mathbb{N}} A(n)$, follows from ${ }^{4}$

$$
\exists^{\text {st }} \alpha \forall^{\text {st }} \varphi, q \exists \beta \in \operatorname{Ap}^{2}(\alpha)(\varphi, q) \forall n \in \operatorname{Ap}(\varphi)(\beta) \forall b \in \operatorname{Ap}(q)(\beta) A_{H}(n, \beta(n), b)
$$

where the types above are

$$
\begin{array}{ll}
\delta: \mathbb{N} \Rightarrow\left(X \Rightarrow \mathcal{P}_{\mathrm{f}}(R)\right) \Rightarrow \mathcal{P}_{\mathrm{f}}(X) & p: X \Rightarrow \mathcal{P}_{\mathrm{f}}(R) \\
q:(\mathbb{N} \rightarrow X) \Rightarrow \mathcal{P}_{\mathrm{f}}(R) & \beta: \mathbb{N} \rightarrow X \\
\varphi:(\mathbb{N} \rightarrow X) \Rightarrow \mathcal{P}_{\mathrm{f}}(\mathbb{N}) & \operatorname{Ap}^{2}(\alpha)(\varphi, q): \mathcal{P}_{\mathrm{f}}(\mathbb{N} \Rightarrow X) .
\end{array}
$$

Given $\delta, \varphi$ and $q$, we will calculate finite sets $\alpha, N$ and $P$ and show that

$$
\begin{aligned}
\forall n \in N & \forall p \in P \exists a \in \operatorname{Ap}^{2}(\delta)(n, p) \forall b \in \operatorname{Ap}(p)(a) A_{H}(n, a, b) \\
& \rightarrow \exists \beta \in \alpha \forall n \in \operatorname{Ap}(\varphi)(\beta) \forall b \in \operatorname{Ap}(q)(\beta) A_{H}(n, \beta(n), b) .
\end{aligned}
$$

Although the Herbrand interpretation here would only actually ask us to produce finite sets of candidate "constructions" for $\alpha, N$ and $P$, with a guarantee that one of them did the job, we show that in fact we can produce concrete finite sets $\alpha, N$ and $P$. Given $\delta, \varphi$ and $q$ as above, let us define

\footnotetext{
${ }^{4}$ Instead of producing a set of functions $\beta: \mathbb{N} \Rightarrow X$ we will actually produce a single function $\beta: \mathbb{N} \rightarrow X$. Note that $\operatorname{Ap}(\{p\})(a)=p(a)$.
} 


$$
\begin{aligned}
& \varepsilon_{n}:\left(X \rightarrow \mathcal{P}_{\mathrm{f}}(R)\right) \rightarrow \mathcal{P}_{\mathrm{f}}(X) \\
& \hat{q}: X^{*} \rightarrow \mathcal{P}_{\mathrm{f}}(R) \\
& \omega:(\mathbb{N} \rightarrow X) \rightarrow \mathbb{N}
\end{aligned}
$$

as

$$
\begin{aligned}
& \varepsilon_{n}(p)=\operatorname{Ap}^{2}(\delta)(n,\{p\}) \\
& \hat{q}(s)=\operatorname{Ap}(q)\left(s^{+}\right) \\
& \omega(\beta)=\max (\operatorname{Ap}(\varphi)(\beta)) .
\end{aligned}
$$

We will then apply hBR to $\varepsilon_{n}, \hat{q}$ and $\omega$.

Theorem 5.2 Define $t=\operatorname{hBR}_{\langle\rangle}^{\omega}(\varepsilon)(\hat{q})$. We claim that

$$
\begin{aligned}
\alpha & =\left\{s^{+}: s \in t\right\} \\
P & =\left\{p_{r}: r \preceq s \wedge s \in t\right\} \\
N & =1+\max \{|s|: s \in t\}
\end{aligned}
$$

where $p_{r}(y)=\bigcup\left\{\hat{q}\left(r * y * r^{\prime}\right): r^{\prime} \in \mathrm{hBR}(r * y)\right\}$, witness the Herbrand interpretation of DNS, i.e.

$$
\begin{aligned}
& \forall n \leq N \forall p \in P \exists a \in \operatorname{Ap}^{2}(\delta)(n, p) \forall b \in \operatorname{Ap}(p)(a) A_{H}(n, a, b) \\
& \rightarrow \exists \beta \in \alpha \forall i \in \operatorname{Ap}(\varphi)(\beta) \forall b \in \operatorname{Ap}(q)(\beta) A_{H}(i, \beta(i), b)
\end{aligned}
$$

viewing the number $N$ as the finite set $\{0,1, \ldots, N\}$.

Proof. Assume

$$
\forall n \leq N \forall p \in P \exists a \in \operatorname{Ap}^{2}(\delta)(n, p) \forall b \in \operatorname{Ap}(p)(a) A_{H}(n, a, b) .
$$

By induction on $n$ it follows that: For all $n \leq N$ there exists a sequence $\left\langle a_{0}, \ldots, a_{n}\right\rangle$ such that either

- for some $i<n, \omega\left(\left\langle a_{0}, \ldots, a_{i}\right\rangle^{+}\right)<i+1$, or

- for all $i \leq n$,

$$
a_{i} \in \underbrace{\operatorname{Ap}^{2}(\delta)\left(i,\left\{p_{\left\langle a_{0}, \ldots, a_{i-1}\right\rangle}\right\}\right)}_{\varepsilon_{i}\left(p_{\left\langle a_{0}, \ldots, a_{i-1}\right\rangle}\right)} \wedge \forall b \in \underbrace{\operatorname{Ap}\left(\left\{p_{\left\langle a_{0}, \ldots, a_{i-1}\right\rangle}\right\}\right)\left(a_{i}\right)}_{p_{\left\langle a_{0}, \ldots, a_{i-1}\right\rangle}\left(a_{i}\right)} A_{H}\left(i, a_{i}, b\right) .
$$

We have used Lemma 4.4 since under the assumption that $\omega\left(\left\langle a_{0}, \ldots, a_{i-1}\right\rangle^{+}\right) \geq i$ for all $i \leq n$ then $\left\langle a_{0}, \ldots, a_{i-1}\right\rangle * r \in t$, for some $r$, and hence $p_{\left\langle a_{0}, \ldots, a_{i-1}\right\rangle} \in P$. By Lemma 4.5 there exists a least $n<N$ such that $\omega\left(\left\langle a_{0}, \ldots, a_{n}\right\rangle^{+}\right)<n+1$, so that (9) holds for all $i \leq n$, and $\left\langle a_{0}, \ldots, a_{n}\right\rangle \in t$. Let $s=\left\langle a_{0}, \ldots, a_{n}\right\rangle$ and $\beta=s^{+}$(so that $\beta \in \alpha$ ). Note that

$$
\max \left(\operatorname{Ap}(\varphi)\left(s^{+}\right)\right)=\omega\left(s^{+}\right)<|s| .
$$


Hence, $i<|s|$ for all $i \in \operatorname{Ap}(\varphi)\left(s^{+}\right)$. By Lemma 4.6

$$
\operatorname{Ap}(q)(\beta)=\operatorname{Ap}(q)\left(s^{+}\right)=\hat{q}(s) \subseteq p_{\left\langle a_{0}, \ldots, a_{i-1}\right\rangle}\left(a_{i}\right), \text { for all } i \in \operatorname{Ap}(\varphi)\left(s^{+}\right) .
$$

By (9) we can conclude that $\forall i \in \operatorname{Ap}(\varphi)(\beta) \forall b \in \operatorname{Ap}(q)(\beta) A_{H}(i, \beta(i), b)$.

A reader familiar with the bounded functional interpretation of DNS (cf. [8]) will have noticed several similarities with the Herbrand functional interpretation of DNS presented here. The main difference, however, is that we have made no effort to formalise the verification of the interpretation in a constructive setting, choosing to view $\neg \forall x \neg A$ as a strong existence $\exists x A$. Although it is clear to us that such formalisation is possible, attempting to do so would complicate the verification and probably obfuscate the crucial steps of the bar recursive construction. We hope that by simplifying the "logical component" of the proof one can better appreciate its "computational" aspect and the use of the "Herbrand" bar recursion. The recent paper [9] sheds some light at the relationship between the two interpretations.

\section{Conclusion}

We conclude by noticing that all lemmas of Section 4 were proven for the specific case of the finite power set monad only. It is reasonable to ask whether more general versions of such lemmas work already for the monadic bar recursion $T$-EPS. The main challenge as we see it is to find the appropriate abstraction to the notion of set containment and subset inclusion. Similarly, one might consider generalisations of the Herbrand functional interpretation whereby the finite power set monads is replaced by an arbitrary monad, with possibly some extra structure.

\section{References}

[1] S. Berardi, M. Bezem, and T. Coquand. On the computational content of the axiom of choice. The Journal of Symbolic Logic, 63(2):600-622, 1998.

[2] U. Berger and P. Oliva. Modified bar recursion. Mathematical Structures in Computer Science, 16:163-183, 2006.

[3] M. Bezem. Strongly majorizable functionals of finite type: a model for bar recursion containing discontinuous functionals. The Journal of Symbolic Logic, 50:652-660, 1985 .

[4] M. H. Escardó and P. Oliva. Computational interpretations of analysis via products of selection functions. In F. Ferreira, B. Lowe, E. Mayordomo, and L. M. Gomes, editors, Computability in Europe 2010, LNCS, pages 141-150. Springer, 2010.

[5] M. H. Escardó and P. Oliva. Selection functions, bar recursion, and backward induction. Mathematical Structures in Computer Science, 20(2):127-168, 2010.

[6] M. H. Escardó and P. Oliva. Sequential games and optimal strategies. Royal Society Proceedings A, 467:1519-1545, 2011. 
[7] M. H. Escardó and P. Oliva. Computational interpretations of analysis via products of selection functions. The Journal of Symbolic Logic, 80(1):1-28, 2015.

[8] F. Ferreira and P. Engrácia. The bounded functional interpretation of the double negation shift. Journal of Symbolic Logic, 75(2):759-773, 2010.

[9] F. Ferreira and J. Gaspar. Nonstandardness and the bounded functional interpretation. Annals of Pure and Applied Logic, 166:665-740, 2015.

[10] K. Gödel. Über eine bisher noch nicht benützte Erweiterung des finiten Standpunktes. Dialectica, 12:280-287, 1958.

[11] A. Kock. Strong functors and monoidal monads. Arch. Math. (Basel), 23:113$120,1972$.

[12] U. Kohlenbach. Effective bounds from ineffective proofs in analysis: an application of functional interpretation and majorization. The Journal of Symbolic Logic, 57:1239-1273, 1992.

[13] E. Moggi. Notions of computation and monads. Inf. Comput., 1:55-92, 1991.

[14] P. Oliva and T. Powell. On Spector's bar recursion. Mathematical Logic Quarterly, 58(4-5):356-365, 2012.

[15] B. Scarpellini. A model for bar recursion of higher types. Compositio Mathematica, 23:123-153, 1971.

[16] C. Spector. Provably recursive functionals of analysis: a consistency proof of analysis by an extension of principles in current intuitionistic mathematics. In F. D. E. Dekker, editor, Recursive Function Theory: Proc. Symposia in Pure Mathematics, volume 5, pages 1-27. American Mathematical Society, Providence, Rhode Island, 1962.

[17] A. S. Troelstra. Metamathematical Investigation of Intuitionistic Arithmetic and Analysis, volume 344 of Lecture Notes in Mathematics. Springer, Berlin, 1973.

[18] Benno van den Berg, Eyvind Martol Briseid, and Pavol Safarik. A functional interpretation for nonstandard arithmetic. Annals of Pure and Applied Logic, 163(2):1962-1994, 2012.

[19] Philip Wadler. The essence of functional programming. In Proceedings of the 19th ACM SIGPLAN-SIGACT Symposium on Principles of Programming Languages, POPL'92, pages 1-14, New York, NY, USA, 1992. ACM. 